OPEN ACCESS

Edited by:

Meng Xie,

Brookhaven National Laboratory, United States

Reviewed by:

Pui Ying Lam,

Kyoto University, Japan Yunjun Zhao,

Brookhaven National Laboratory (DOE), United States

*Correspondence: Ralf Stracke

ralf.stracke@uni-bielefeld.de

Specialty section: This article was submitted to

Plant Metabolism and

Chemodiversity,

a section of the journal

Frontiers in Plant Science

Received: 28 April 2021 Accepted: 20 July 2021 Published: 17 August 2021

Citation:

Busche M, Acatay C, Martens S, Weisshaar B and Stracke R (2021)

Functional Characterisation of Banana (Musa spp.) 2-Oxog/utarateDependent Dioxygenases Involved in

Flavonoid Biosynthesis.

Front. Plant Sci. 12:701780.

doi: 10.3389/fpls.2021.701780

\section{Functional Characterisation of Banana (Musa spp.) 2-Oxoglutarate- Dependent Dioxygenases Involved in Flavonoid Biosynthesis}

\author{
Mareike Busche ${ }^{1}$, Christopher Acatay ${ }^{1}$, Stefan Martens ${ }^{2}$, Bernd Weisshaar ${ }^{1}$ and \\ Ralf Stracke ${ }^{1 *}$
}

'Genetics and Genomics of Plants, Faculty of Biology, Bielefeld University, Bielefeld, Germany, ${ }^{2}$ Fondazione Edmund Mach, Research and Innovation Centre, San Michele All' Adige, Italy

Bananas (Musa) are non-grass, monocotyledonous, perennial plants that are well known for their edible fruits. Their cultivation provides food security and employment opportunities in many countries. Banana fruits contain high levels of minerals and phytochemicals, including flavonoids, which are beneficial for human nutrition. To broaden the knowledge on flavonoid biosynthesis in this major crop plant, we aimed to identify and functionally characterise selected structural genes encoding 2-oxoglutarate-dependent dioxygenases, involved in the formation of the flavonoid aglycon. Musa candidates genes predicted to encode flavanone 3-hydroxylase (F3H), flavonol synthase (FLS) and anthocyanidin synthase (ANS) were assayed. Enzymatic functionalities of the recombinant proteins were confirmed in vivo using bioconversion assays. Moreover, transgenic analyses in corresponding Arabidopsis thaliana mutants showed that MusaF3H, MusaFLS and MusaANS were able to complement the respective loss-of-function phenotypes, thus verifying functionality of the enzymes in planta. Knowledge gained from this work provides a new aspect for further research towards genetic engineering of flavonoid biosynthesis in banana fruits to increase their antioxidant activity and nutritional value.

Keywords: banana, specialised metabolites, flavanone 3-hydroxylases, flavonol synthase, anthocyanidin synthase

\section{INTRODUCTION}

Banana (Musa spp.) plants are well known for their edible fruit and serve as a staple food crop in Africa, Central and South America (Arias et al., 2003). With more than 112 million tons produced in 2016, bananas are among the most popular fruits in the world and provide many employment opportunities (FAO, 2019). Furthermore, banana fruits are rich in health promoting minerals and phytochemicals, including flavonoids, a class of plant specialised metabolites, which contribute to the beneficial effects through their antioxidant characteristics (Forster et al., 2003; Wall, 2006; Singh et al., 2016). Flavonoid molecules share a C6-C3-C6 aglycon core, which can be reorganised or modified, e.g. by oxidation or glycosylation (Tanaka et al., 2008; Le Roy et al., 2016). Modifications at the central ring structure divide flavonoids into 10 major subgroups (i.e. chalcones, aurones, flavanones, flavones, isoflavones, dihydroflavonols, 
flavonols, leucoanthocyanidins, anthocyanidins and flavan-3-ols). The diversity in chemical structure is closely related to diverse bioactivities of flavonoids in plant biology and human nutrition (Falcone Ferreyra et al., 2012). For example, anthocyanins are in many cases well known to colour flowers and fruits to attract animals and thus promoting pollination and dispersion of seeds (Ishikura and Yoshitama, 1984; Gronquist et al., 2001; Grotewold, 2006). Flavonols can interact with anthocyanins to modify the colour of fruits (Andersen and Jordheim, 2010) and play a prominent role in protection against UV-B irradiation (Li et al., 1993) and also in plant fertility (Mo et al., 1992).

Many researchers have attributed positive effects on human health to flavonoids: e.g. antigenotoxic (Dauer et al., 2003), anticarcinogenic and antioxidative (Kandil et al., 2002) effects, as well as the prevention of cardiovascular diseases has been suggested (summarised in Perez-Vizcaino and Duarte, 2010). Additionally, Sun et al. (2019) suggested an involvement of flavonoids in the plant defence against the tropical race 4 (TR4) of the Musa Fusarium wilt (commonly known as 'panama disease') pathogen Fusarium oxysporum f. sp. cubense (Foc), which is a threat to the global banana production. It is certainly interesting to take a closer look at the biosynthesis of flavonoids in Musa.

Flavonoids are derived from the amino acid L-phenylalanine and malonyl-coenzyme A. Their biosynthesis (Figure 1) has been analysed in many different species including the dicotyledonous model plant Arabidopsis thaliana (Hahlbrock and Scheel, 1989; Lepiniec et al., 2006) and the monocotyledonous crop plants Zea mays (summarised in Tohge et al., 2017) and Oryza sativa (summarised in Goufo and Trindade, 2014). The first committed step, catalysed by the enzyme chalcone synthase (CHS), is the formation of naringenin chalcone from $p$-coumaroyl CoA and malonyl CoA (Kreuzaler and Hahlbrock, 1972). A heterocyclic ring is introduced during the formation of naringenin (a flavanone) from naringenin chalcone, which can occur spontaneously or catalysed by chalcone isomerase (CHI; Bednar and Hadcock, 1988). The enzyme flavanone 3-hydroxylase (F3H or FHT) converts flavanones to dihydroflavonols by hydroxylation at the C-3 position (Forkmann et al., 1980). Alternatively, flavanones can be converted to flavones by flavone synthase I or II (FNSI or II; Britsch, 1990). Flavonol synthase (FLS) catalyses the conversion of dihydroflavonols to the corresponding flavonols by introducing a double bond between C-2 and C-3 (Forkmann et al., 1986; Holton et al., 1993). Moreover, dihydroflavonols can be converted to leucoanthocyanidins by dihydroflavonol 4-reductase (DFR; Heller et al., 1985), which competes with FLS for substrates (Luo et al., 2016). Anthocyanidin synthase (ANS; also termed leucoanthocyanidin dioxygenase, LDOX) converts leucoanthocyanidins to anthocyanidins (Saito et al., 1999).

The flavonoid biosynthesis enzymes belong to different functional classes (summarised in Winkel, 2006): polyketide synthases (e.g. CHS), 2-oxoglutarate-dependent dioxygenases (2-ODD; e.g. F3H, ANS, FLS and FNSI), short-chain dehydrogenases/reductases (e.g. DFR), aldo-keto reductases (e.g. chalcone reductase, $\mathrm{CHR}$ ) and cytochrome $\mathrm{P} 450$ monooxygenases (e.g. FNSII and F3'H). Flavonoid biosynthesis is evolutionary old in plants (Wen et al., 2020) and, although differences exist, quite similar in dicots like A. thaliana and monocots-like Musa.

In the present study, we focus on the functional class of flavonoid aglycon forming enzymes, namely, the 2-ODDs. 2-ODDs occur throughout the kingdom of life and play important roles in many biological processes including oxygen sensing and DNA repair (Jaakkola et al., 2001; Trewick et al., 2002). They are a class of non-heme iron-containing enzymes, which require 2-oxoglutarate, $\mathrm{Fe}^{2+}$ and ascorbate for substrate conversion (summarised in Prescott and John, 1996).

Plant 2-ODDs share conserved amino acid residues which coordinate ferrous iron binding $(\mathrm{HxDxnH})$ and binding of 2-oxoglutarate (RxS; Cheng et al., 2014). They can be divided into three distinct evolutionary classes, DOXA, DOXB and DOXC, based on amino acid similarity (Kawai et al., 2014). The 2-ODDs involved in specialised metabolism were classified into the DOXC class. Yet, all flavonoid biosynthesis related 2-ODDs were found in the DOXC subclades 28 (F3H and FNSI) or 47 (FLS and ANS; Kawai et al., 2014). In some cases, the high amino acid similarity between the different enzymes leads to overlapping functions. For example, FLSs from Ginkgo biloba (Xu et al., 2012) and O. sativa (Park et al., 2019) can accept flavanones and dihydroflavonols as substrates. Also, ANSs from A. thaliana (Stracke et al., 2009) and Malus domestica (Yan et al., 2005) can catalyse the formation of flavonol glycosides.

Based on the Musa acuminata genome sequence annotation (DHont et al., 2012; Pandey et al., 2016) identified 28 putative Musa flavonoid biosynthesis enzymes. This includes seven 2-ODD-type enzymes, namely, two $\mathrm{F} 3 \mathrm{H}$, four FLS and one ANS, while no suitable FNSI candidate was identified. While the expression of the respective genes and correlations of expression to flavonoid metabolite accumulation have been studied, the functionality of these enzymes has not been analysed until now.

Here, we describe the sequence-based and functional characterisation of 2-ODDs from the non-grass monocot Musa. Functionalities of recombinant Musa enzymes were analysed using in vivo bioconversion assays and by in planta complementation of corresponding A. thaliana loss-of-function mutants. This resulted in the experimental confirmation that the Musa flavonoid 2-ODDs studied have the predicted functions. The presented results contribute to the understanding of flavonoid biosynthesis in Musa. They provide a strong basis for further research to enhance the efficiency of flavonoid production in banana, in order to increase the fruits' health promoting effects.

\section{MATERIALS AND METHODS}

\section{Plant Material}

Banana plants (Grand Naine, plantain) for RNA extraction were grown in the field in Lucknow, India. Musa gene annotation identifiers refer to the study of Martin et al. (2016). Columbia-0 (Col-0, NASC ID N1092) and Nössen-0 (Nö-0, NASC ID N3081) were used as wild-type controls. The A. thaliana mutants tt6-2 ( 3 hh, GK-292E08, Col-0 background; Appelhagen et al., 2014) 


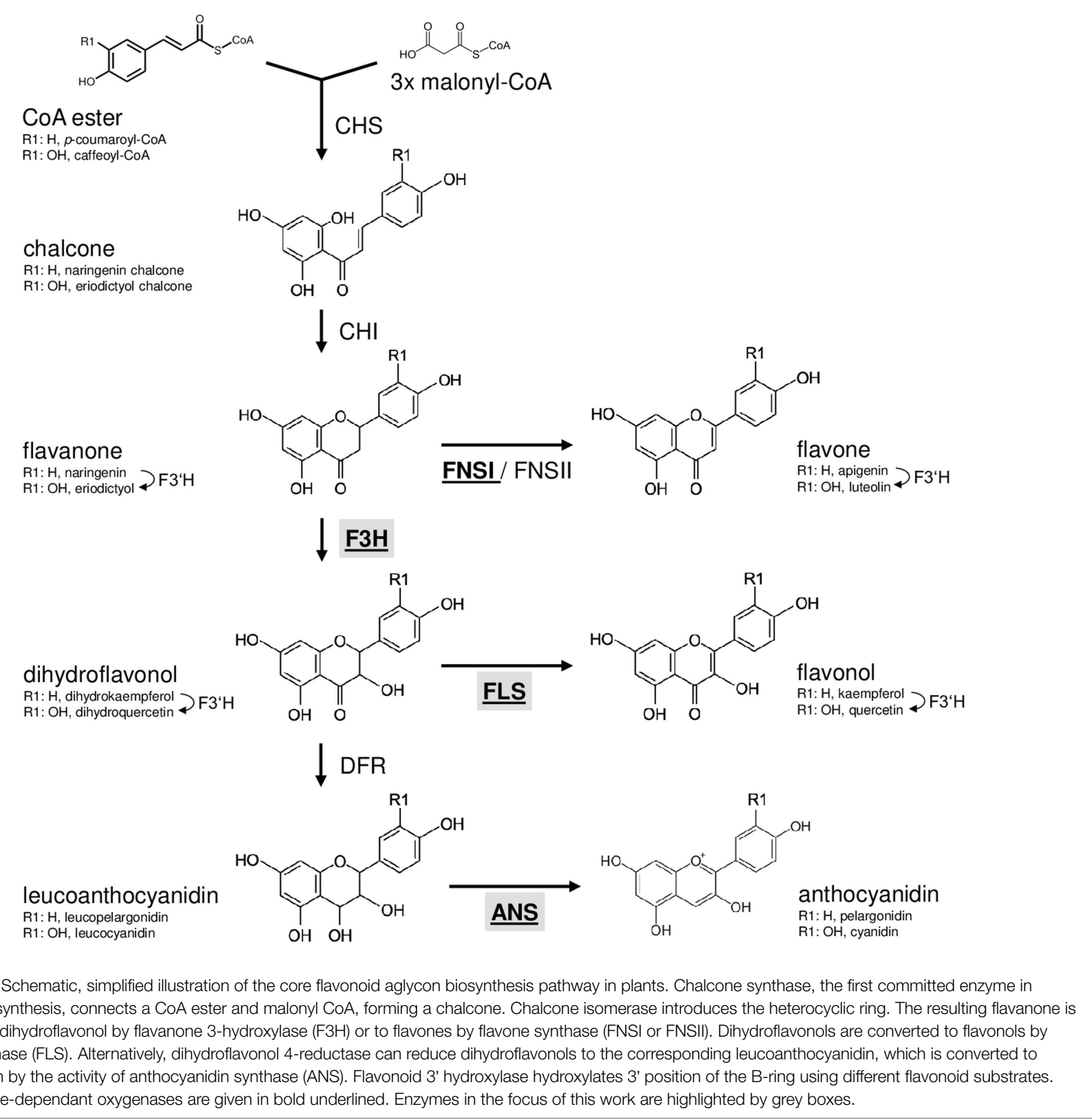

and ans/fls1-2 (synonym ldox/fls1-2, ldox: SALK_028793, Col-0 background; fls1-2: RIKEN_PST16145, Nö-0 background; Stracke et al., 2009) were used for complementation experiments.

\section{Phylogenetic Analysis}

Multiple protein sequence alignments were created with MAFFT v7 (Katoh and Standley, 2013) using default settings. The approximately maximum-likelihood phylogenetic tree of 33 plant 2-ODDs with proven F3H, FNSI, FLS and ANS functionality (Supplementary Table S1) and seven Musa 2-ODDs was constructed as described by Pucker et al. (2020b): MAFFT alignments were cleaned with pxclsq (Brown et al., 2017) and the tree was constructed with FastTree v2.1.10 using the WAG+CAT model (Price et al., 2010). The tree was visualised with interactive tree of life (Letunic and Bork, 2019), branch lengths were neglected.

\section{Expression Analysis}

Expression data for Musa 2-ODD genes were extracted from previous analyses (Pucker et al., 2020a). Short Read Archive IDs can be found in Supplementary Table S2.

\section{Total RNA Extraction, cDNA Synthesis and Molecular Cloning}

Isolation of RNA from different Musa plant organs (leaf, pseudostem, bract, fruit peel and fruit pulp) was performed according to a protocol from Asif et al. (2000). cDNA synthesis was performed from $1 \mu \mathrm{g}$ total RNA using the ProtoScript ${ }^{\circledR}$ 
First Strand cDNA Synthesis Kit [New England Biolabs, (NEB)] with the provided random primer mix according to the suppliers' instructions. Amplification of predicted full-length CDSs (Pandey et al., 2016) were done using Q $5^{\circledR}$ High-Fidelity DNA polymerase (NEB) and gene-specific primers (Supplementary Table S3) according to standard protocols. Creation of full-length coding sequence constructs (CDS) was performed using the GATEWAY ${ }^{\circledR}$ Technology (Invitrogen). MusaF3H1 (Ma02_t04650), MusaF3H2 (Ma07_t17200), MusaFLS1 (Ma03_t06970), MusaFLS3 (Ma10_ t25110) and MusaANS (Ma05_t03850) CDSs were successfully amplified on different cDNA pools. The resulting PCR products were recombined into $\mathrm{pDONR}^{\mathrm{TM}} / \mathrm{Zeo}$ (Invitrogen) with $\mathrm{BP}$ clonase (Invitrogen) resulting in Entry plasmids, which were sequenced by Sanger technology (Sanger et al., 1977) on 3730XL sequencers using BigDye terminator v3.1 chemistry (Thermo Fisher). Entry plasmids for AtF3H, AtFLS1 and AtMYB12 were available from previous studies (Preuss et al., 2009; Stracke et al., 2017), Petroselinum crispum FNSI (PcFNSI) was amplified on a plasmid from a previous study (Martens et al., 2003). The full-length CDSs were introduced from the Entry plasmids into the inducible Escherichia coli expression vector pDEST17 (Invitrogen) and the binary expression vector pLEELA (Jakoby et al., 2004) using GATEWAY LR reaction (Invitrogen).

\section{Heterologous Expression in E. coli}

pDEST17-based plasmids containing proT7-RBS-6xHis-CDST7term expression cassettes (proT7: T7 promoter, RBS: ribosomebinding site, 6xHis: polyhistidine tag and T7term: T7 transcription terminator) were transformed into BL21-AI cells (Invitrogen). Cultures were grown in $\mathrm{LB}$ to an $\mathrm{OD}_{600}$ of about 0.4 and expression was induced with $0.2 \% \mathrm{~L}$-arabinose.

\section{F3H and FLS Bioconversion Assay in E. coli}

The enzyme assay was performed using $20 \mathrm{ml}$ E. coli cultures expressing the respective constructs right after induction with L-arabinose. $100 \mu \mathrm{l}$ substrate $[10 \mathrm{mg} / \mathrm{ml}$ naringenin, eriodictyol or dihydroquercetin (DHQ)], $50 \mu \mathrm{l}$ 2-oxoglutaric acid, $50 \mu \mathrm{l}$ $\mathrm{FeSo}_{4}$ and $50 \mu \mathrm{l} 1 \mathrm{M}$ sodium ascorbate were added. The cultures were incubated at $28^{\circ} \mathrm{C}$ overnight. To extract flavonoids, $1 \mathrm{ml}$ was removed from each culture and mixed with $200 \mu$ l ethyl acetate by vortexing for $30 \mathrm{~s}$. Samples were taken after $0 \mathrm{~h}$, $1 \mathrm{~h}, 2 \mathrm{~h}, 3 \mathrm{~h}, 4 \mathrm{~h}$ and $24 \mathrm{~h}$. After centrifugation for $2 \mathrm{~min}$ at $14,000 \mathrm{~g}$, the organic phase was transferred into a fresh reaction tube. Flavonoid content was analysed by highperformance thin-layer chromatography (HPTLC). Naringenin (Sigma), dihydrokaempferol (DHK; Sigma), kaempferol (Roth), eriodictyol (TransMIT PlantMetaChem), apigenin (TransMIT PlantMetaChem), DHQ (Roth) and quercetin (Sigma) were dissolved in methanol and used as standards. $3 \mu \mathrm{l}$ of each methanolic extract was spotted on a HPTLC Silica Gel 60 plate (Merck). The mobile phase was composed of chloroform, acetic acid and water mixed in the ratio (50:45:5). Flavonoid compounds were detected as described previously (Stracke et al., 2007), using diphenylboric acid 2-aminoethyl ester (DPBA) and UV light (Sheahan and Rechnitz, 1992).

\section{Agrobacterium-Mediated Transformation of $A$. thaliana}

T-DNA from pLEELA-based plasmids containing 2xpro35S-driven Musa2-ODDs was transformed into $A$. thaliana plants via Agrobacterium tumefaciens [Agrobacterium, GV101::pMP90RK, (Koncz and Schell, 1986)] mediated gene transfer using the floral dip method (Clough and Bent, 1998). Successful T-DNA integration was verified by BASTA selection and PCR-based genotyping.

\section{Flavonoid Staining of $A$. thaliana Seedlings}

In situ visualisation of flavonoids in norflurazon-bleached A. thaliana seedlings was performed according to Stracke et al. (2007) using DPBA/Triton X-100 and epifluorescence microscopy.

\section{Analysis of Flavonols in Methanolic Extracts}

Flavonol glycosides were extracted and analysed as previously described (Stracke et al., 2009). A. thaliana rosette leaves were homogenised in $80 \%$ methanol, incubated for $15 \mathrm{~min}$ at $70^{\circ} \mathrm{C}$ and centrifuged for $10 \mathrm{~min}$ at $14,000 \mathrm{~g}$. Supernatants were vacuum dried. The dried pellets were dissolved in $80 \%$ methanol and analysed by HPTLC on a Silica Gel 60 plate (Merck). Ethyl acetate, formic acid, acetic acid and water (100:26:12:12) were used as a mobile phase. Flavonoid compounds were detected as described above.

\section{Determination of Anthocyanin Content}

To induce anthocyanin production, A. thaliana seedlings were grown on $0.5 \mathrm{MS}$ plates with $4 \%$ sucrose and $16 \mathrm{~h}$ of light illumination per day at $22^{\circ} \mathrm{C}$. Six-day-old seedlings were used to photometrically quantify anthocyanins as described by Mehrtens et al. (2005). All samples were measured in three independent biological replicates. Error bars indicate the standard error of the average anthocyanin content. Statistical analysis was performed using the Mann-Whitney $U$ test (Mann and Whitney, 1947).

\section{RESULTS}

\section{Creation of cDNA Constructs and Sequence-Based Characterisation of Musa Flavonoid 2-ODDs}

Previously described putative Musa flavonoid biosynthesis enzymes encoded in the $M$. acuminata, Pahang $\mathrm{DH}$ reference genome sequence were used. This included two $\mathrm{F} 3 \mathrm{Hs}$, four FLSs and one ANS, which were classified as 2-ODDs. To functionally characterise these Musa enzymes, we amplified the corresponding CDSs from a cDNA template collection derived from different $M u s a$ organs, using primers designed on the Pahang DH reference genome sequence. The successfully amplified cDNAs of MusaF3H1 and MusaF3H2 were derived from plantain pulp, MusaFLS1 from Grand Naine young leaf, MusaFLS3 on Grand Naine bract and MusaANS on Grand Naine peel. Unfortunately, we were not able to amplify MusaFLS2 and MusaFLS4 from our template collection. 
Comparison of the resulting 2-ODD cDNA sequences with the reference sequence revealed several single-nucleotide polymorphisms (Supplementary File S1). The derived amino acid sequences show close similarity to other plant 2-ODD proteins known to be involved in flavonoid biosynthesis (Supplementary File S2). The amino acids well known to coordinate ferrous iron binding $(\mathrm{HxDxnH})$ and binding of 2-oxoglutarate (RxS) are also conserved in the Musa 2-ODDs. Additionally, the AtFLS1 residues which have been shown to be involved in flavonoid substrate binding (Supplementary File S2) are conserved. The residue F293 (all positions refer to AtFLS1) is conserved in all Musa 2-ODD proteins, F134 and K202 are found in the MusaFLSs and MusaANS and E295 is conserved in MusaANS.

To analyse the evolutionary relationship between Musa 2-ODDs and 33 known flavonoid biosynthesis-related 2-ODDs, a phylogenetic tree was built (Figure 2). The phylogenetic tree revealed two distinct clades, which correspond to the DOXC28 and DOXC47 classes of the 2-ODD superfamily. In the F3Hand FNSI-containing DOXC28 class, $M u s a \mathrm{~F} 3 \mathrm{H} 1$ and $M u s a \mathrm{~F} 3 \mathrm{H} 2$ cluster with other $\mathrm{F} 3 \mathrm{Hs}$ from monocotyledonous plants. In the FLS- and ANS-containing DOXC47 class, the MusaANS clusters with ANSs from monocotyledonous plants, while the FLSs from monocotyledonous plants do not form a distinct group, although the MusaFLSs are in proximity to ZmFLS and OsFLS.

Our analyses clearly show that the CDSs identified for MusaF3H1, MusaF3H2, MusaFLS1, MusaFLS3 and MusaANS have the potential to encode functional flavonoid 2-ODDs.

\section{Expression Profiles of Musa 2-ODD Genes}

Analysis of the expression patterns of the genes studied was performed using published RNA-Seq data. We obtained normalised RNA-Seq read values for Musa 2-ODD genes from several organs and developmental stages (Table 1). MusaF3H1 and $\mathrm{MusaF} 3 \mathrm{H} 2$ are expressed in almost all analysed organs and developmental stages. MusaF3H1 expression is highest in early developmental stages of pulp (S1 and S2), followed by intermediate developmental stages of peel (S2 and S3). MusaF3H2 shows highest expression in adult leaves. MusaFLS2 was not expressed in any of the analysed samples. All four MusaFLS genes show low or no expression in seedlings and embryogenic cell suspension. MusaFLS1 shows variance in transcript abundance with particularly high levels in peel (S2) and in young and adult leaves. MusaFLS3 and MusaFLS4 transcript levels are comparatively constant and low, with highest transcript abundance in root and peel ( $\mathrm{S} 1$ and $\mathrm{S} 3$, respectively). While MusaFLS1 transcript abundance is highest in adult leaf, MusaFLS3 and MusaFLS4 lack expression in this tissue. MusaANS shows highest transcript abundance in pulp (S1, S2 and S4) and peel (S3) and very low expression in embryogenic cell suspension, seedlings and leaves.

\section{MusaF3H1 and MusaF3H2 Are Functional F3H}

To confirm $\mathrm{F} 3 \mathrm{H}$ activity in vivo, we used a bioconversion assay. $\mathrm{MusaF} 3 \mathrm{H} 1$ and $\mathrm{MusaF} 3 \mathrm{H} 2$ were heterologously expressed in E. coli and the bacterial cultures were fed with naringenin or eriodictyol as substrate of $\mathrm{F} 3 \mathrm{H}$. Both recombinant proteins, Musa $\mathrm{F} 3 \mathrm{H} 1$ and $M u s a \mathrm{~F} 3 \mathrm{H} 2$, were able to convert naringenin to $\mathrm{DHK}$ in the presence of 2-oxoglutarate and ferrous iron (Figure 3A). After $24 \mathrm{~h}$ of incubation, only small amounts of naringenin remained un-converted. Conversion of the formed $\mathrm{DHK}$ to kaempferol by $M u s a \mathrm{~F} 3 \mathrm{H} 1$ or $M u s a \mathrm{~F} 3 \mathrm{H} 2$ was not observed. Furthermore, eriodictyol was converted to DHQ by MusaF3H1 and MusaF3H2 (Supplementary File S3). A further conversion to the quercetin was not observed. Since $M u s a \mathrm{~F} 3 \mathrm{H} 2$ was previously considered as FNSI candidate, we analysed FNSI activity in a bioconversion assay. While MusaF3H2 was able to convert naringenin to $\mathrm{DHK}$ we detected apigenin as product only for PcFNSI but not for MusaF3H2 (Supplementary File S4). Accordingly, MusaF3H2 does not show FNSI activity in our assay. For further in planta analysis, we chose a complementation assay with an $A$. thaliana $f 3 h$ mutant (tt6-2) which expresses FLS and ANS but no F3H. MusaF3H1 or MusaF3H2 were expressed in $t t 6-2$ plants under the control of the constitutive $2 \times 35 S$ promoter. The accumulation of flavonol glycosides was analysed in herbizide-bleached seedlings using DPBA staining (Figure 3B). While Col-0 wild-type seedlings appeared yellow under UV light, indicating the accumulation of flavonol glycosides, tt6-2 seedlings showed a red fluorescence. $t t 6-2$ mutants expressing MusaF3H1 or MusaF3H2 were able to complement the mutant phenotype, showing the characteristic yellow flavonol glycoside fluorescence of the wild type. These results demonstrate that $M u s a \mathrm{~F} 3 \mathrm{H} 1$ and $M u s a \mathrm{~F} 3 \mathrm{H} 2$ are functional enzymes with $\mathrm{F} 3 \mathrm{H}$ activity and are able to catalyse the conversion of naringenin to $\mathrm{DHK}$.

\section{MusaFLS1 and MusaFLS3 Are Functional FLS}

To confirm FLS activity in vivo, the enzymes were assayed in a coupled bioconversion experiment (Figure 4A). Two E. coli cultures expressing MusaF3H1 and a MusaFLS were mixed and fed with naringenin in the presence of 2-oxoglutarate and ferrous irons. Here, MusaF3H1 converts naringenin to DHK, thus providing the substrate for the FLS enzyme to be tested. While MusaFLS1 was found to be able to convert DHK to kaempferol under the assay conditions, MusaFLS3 was not. In a second bioconversion experiment, E. coli cultures expressing MusaFLS1 or MusaFLS3 were fed with DHQ (Supplementary File S5). Again, MusaFLS1 was able to convert the dihydroflavonol to the corresponding flavonol, while MusaFLS3 was not. We checked the expression of recombinant MusaFLS1 and MusaFLS3 in the E. coli cultures by SDS-PAGE and found both MusaFLS proteins being expressed at similar levels (Supplementary File S7). Moreover, we analysed a possible F3H/FLS biofunctional activity of MusaFLS1 and MusaFLS3 by feeding naringenin to $E$. coli cultures. While MusaFLS1 was not able to convert naringenin to DHK in the assay, MusaFLS3 showed F3H activity but not a further conversion to kaempferol (Supplementary File S6). To further analyse the enzymatic properties in planta, MusaFLS1 or MusaFLS3 was expressed in the flavonol and anthocyanin-deficient 


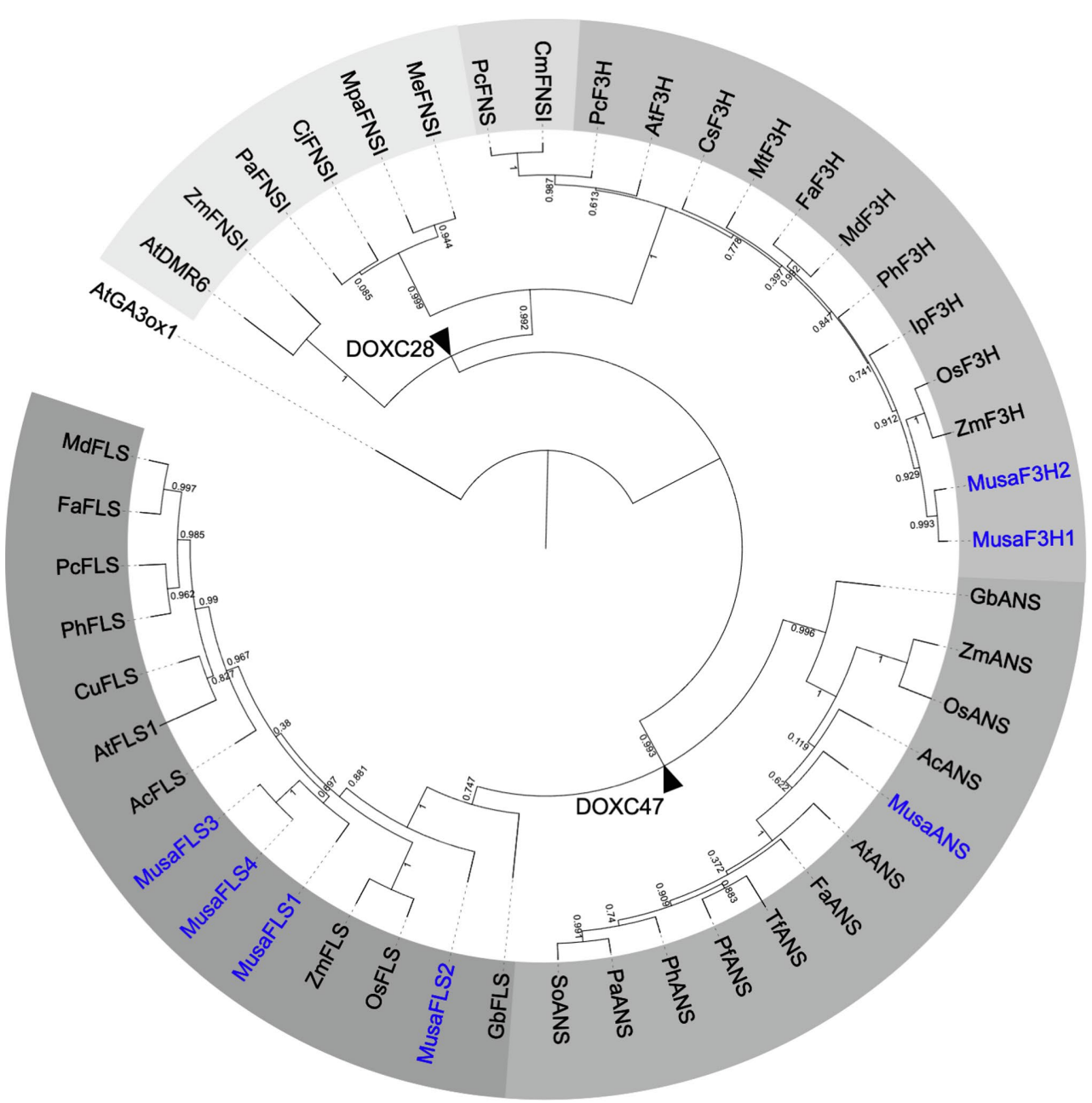

FIGURE 2 | Rooted approximately maximum-likelihood (ML) phylogenetic tree of 2-ODDs involved in flavonoid biosynthesis. ODDs from banana are given in blue, 39 enzymes with proven F3H, FNSI, FLS or ANS activity were included. Different grey scales indicate F3H, FLS, ANS and two evolutionary FNSI clades. AtGA3ox1 (gibberellin 3 beta-hydroxylase1) was used as an outgroup. Branch points to DOXC28 and DOXC47 classes are marked with black arrowheads.

A. thaliana ans/fls1-2 double mutant. HPTLC analyses of methanolic extracts from rosette leaves from greenhouse-grown plants (Figure 4B) showed that wild-type plants (Col-0 and Nö-0) contained flavonol glycosides, including the prominent derivatives kaempferol 3-O-rhamnoside-7-O-rhamnoside (K-3R7R), kaempferol 3-O-glucoside-7-O-rhamnoside (K-3G-7R) and kaempferol 3[-O-rhamnosyl-glucoside]-7-O-rhamnoside (K-3[G$\mathrm{R}]-7 \mathrm{R})$. ans/fls1-2 plants accumulated several dihydroflavonols but did not show flavonol derivatives. ans/fls1-2 mutants transformed with $2 \times 35 S:: M u s a F L S 1$ or $2 \times 35 S:: M u s a F L S 3$ constructs were able to form several flavonol derivatives. Nevertheless, intensities and accumulation patterns of flavonol glycosides varied. We also analysed if MusaFLS1 and MusaFLS3 are able to complement the anthocyanin deficiency of the ans/ fls1-2 double mutant. For this, seedlings were grown on $4 \%$ sucrose to induce anthocyanin accumulation. As shown in Figure 4C, 6-day-old Col-0 and Nö-0 seedlings were able to accumulate red anthocyanin pigments, while ans/fls1-2 transformed with MusaFLS1 or MusaFLS3 did not show visible anthocyanins. These results confirm that MusaFLS1 and MusaFLS3 are functional proteins with FLS activity and are enzymes able to catalyse the conversion of dihydroflavonol to flavonol.

\section{MusaANS Is a Functional ANS}

We analysed MusaANS functionality in a complementation assay with the ans/fls1-2 A. thaliana double mutant. To examine the ability of MusaANS to complement the ans/fls1-2 anthocyanin deficiency phenotype, seedlings were grown on anthocyanininducing media. Anthocyanin accumulation was analysed visually and quantified photometrically (Figures 5A,B). While wild-type seedlings showed red pigmentation, the ans/fls1-2 seedlings did not. ans/fls1-2 seedlings expressing MusaANS showed accumulation of anthocyanins. The anthocyanin content in the complemented seedlings was strongly increased compared to ans/fls1-2 knockout plants, indicating ANS activity. Furthermore, we analysed the 
TABLE 1 | Expression profiles of Musa2-ODD genes in different organs and developmental stages based on RNA-Seq data.

\begin{tabular}{|c|c|c|c|c|c|c|c|c|c|c|c|c|c|c|c|c|c|}
\hline \multirow[b]{2}{*}{ Gene } & \multirow[b]{2}{*}{ 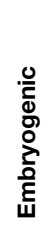 } & \multirow[b]{2}{*}{ 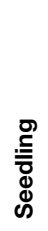 } & \multirow[b]{2}{*}{$\begin{array}{l}\stackrel{+}{\circ} \\
\stackrel{0}{\pi}\end{array}$} & \multicolumn{4}{|c|}{ Leaf } & \multicolumn{5}{|c|}{ Pulp } & \multicolumn{5}{|c|}{ Peel } \\
\hline & & & & Фٓ & 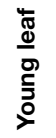 & 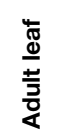 & $\begin{array}{l}\frac{\bar{\Phi}}{\frac{\pi}{\Phi}} \\
\frac{\bar{\sigma}}{0}\end{array}$ & $\frac{ㅇ ㅡ ㄱ ~}{2}$ & $\begin{array}{l}\bar{\infty} \\
\text { 음 } \\
\text { a }\end{array}$ & $\begin{array}{l}\text { N } \\
\text { 음 } \\
\text { Q }\end{array}$ & $\begin{array}{l}\text { ஜ } \\
\text { 음 } \\
\text { a }\end{array}$ & 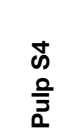 & $\begin{array}{l}\bar{\Phi} \\
\Phi\end{array}$ & $\begin{array}{c}\bar{\omega} \\
\bar{\Phi} \\
\Phi\end{array}$ & $\begin{array}{l}\text { ऊ } \\
\frac{0}{\Phi} \\
\Phi\end{array}$ &  & $\begin{array}{l}\text { ळ } \\
\frac{\Phi}{\Phi} \\
\Omega\end{array}$ \\
\hline MusaF3H2 & 21 & 0 & 23 & 57 & 39 & 171 & 7 & 32 & 96 & 14 & 3 & 14 & 19 & 7 & 27 & 33 & 9 \\
\hline MusaFLS1 & 6 & 0 & 4 & 189 & 91 & 643 & 16 & 2 & 2 & 1 & 2 & 2 & 29 & 3 & 99 & 7 & 7 \\
\hline MusaFLS2 & 0 & 0 & 0 & 0 & 0 & 0 & 0 & 0 & 0 & 0 & 0 & 0 & 0 & 0 & 0 & 0 & 0 \\
\hline MusaANS & 1 & 3 & 90 & 41 & 79 & 3 & 1 & 255 & 559 & 298 & 26 & 137 & 73 & 47 & 50 & 163 & 30 \\
\hline
\end{tabular}

Expression levels are given in fragments per kilo base per million mapped reads. Different grey scales indicate expression levels with higher levels given in darker grey. S1-S4 indicate stages of pulp and peel developments from immature to ripe (Lu et al., 2018).

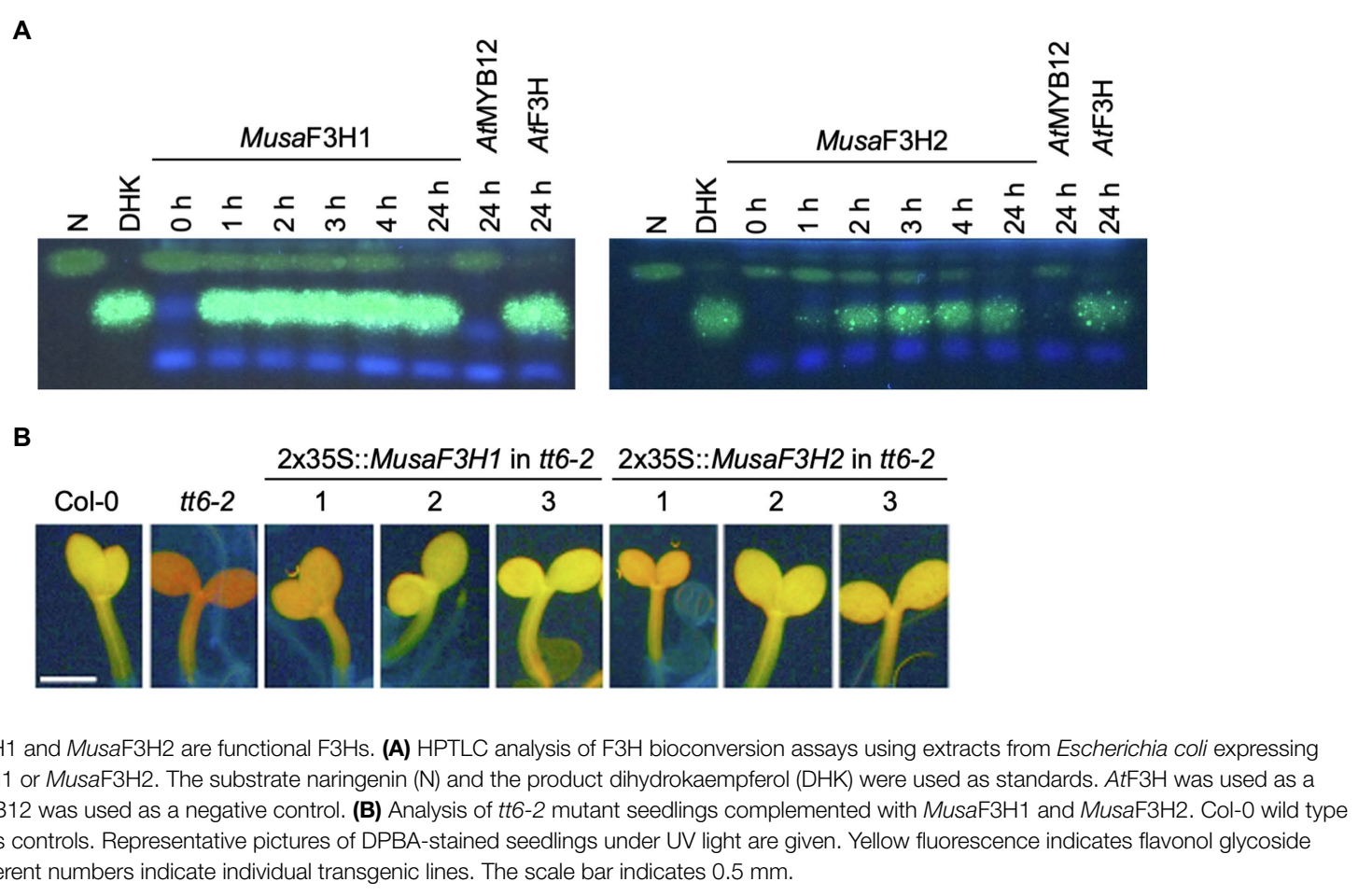

ability of MusaANS to complement the flavonol deficiency phenotype of ans/fls1-2 plants. For this, methanolic extracts of seedlings were analysed by HPTLC followed by DPBA staining (Figure 5C). While wild-type seedlings accumulated several kaempferol and quercetin glycosides, ans/fls1-2 mutants expressing MusaANS showed a flavonoid pattern identical to the ans/fls1-2 mutant, accumulating dihydroflavonol derivatives, but no flavonol derivatives. These results indicate that MusaANS is a functional enzyme with ANS activity and is able to catalyse the conversion of leucoanthocyanidin to anthocyanidin. 
A
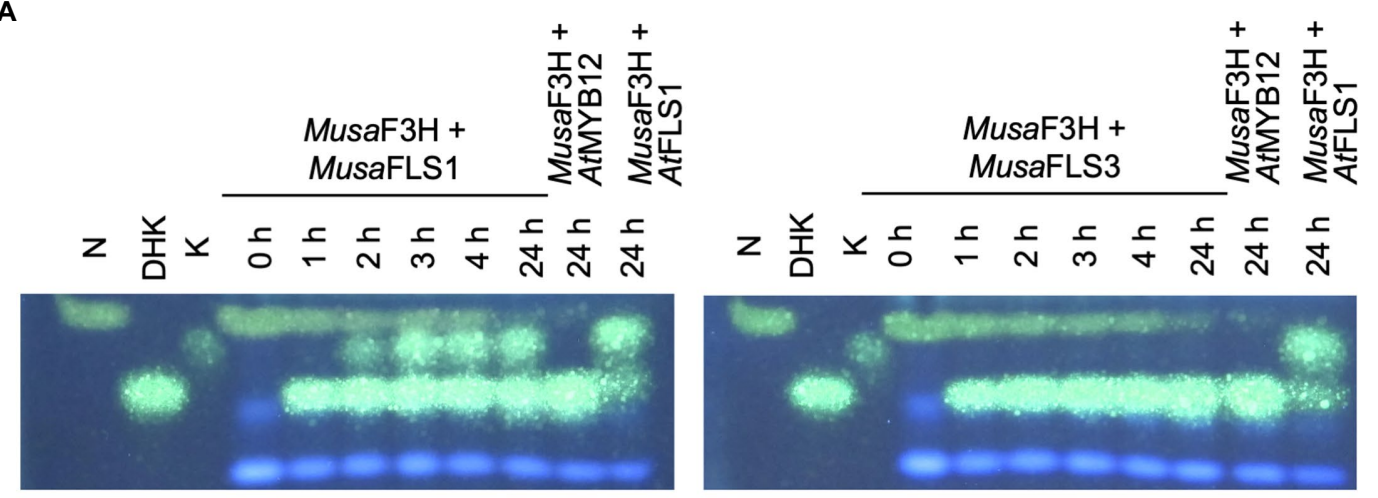

B
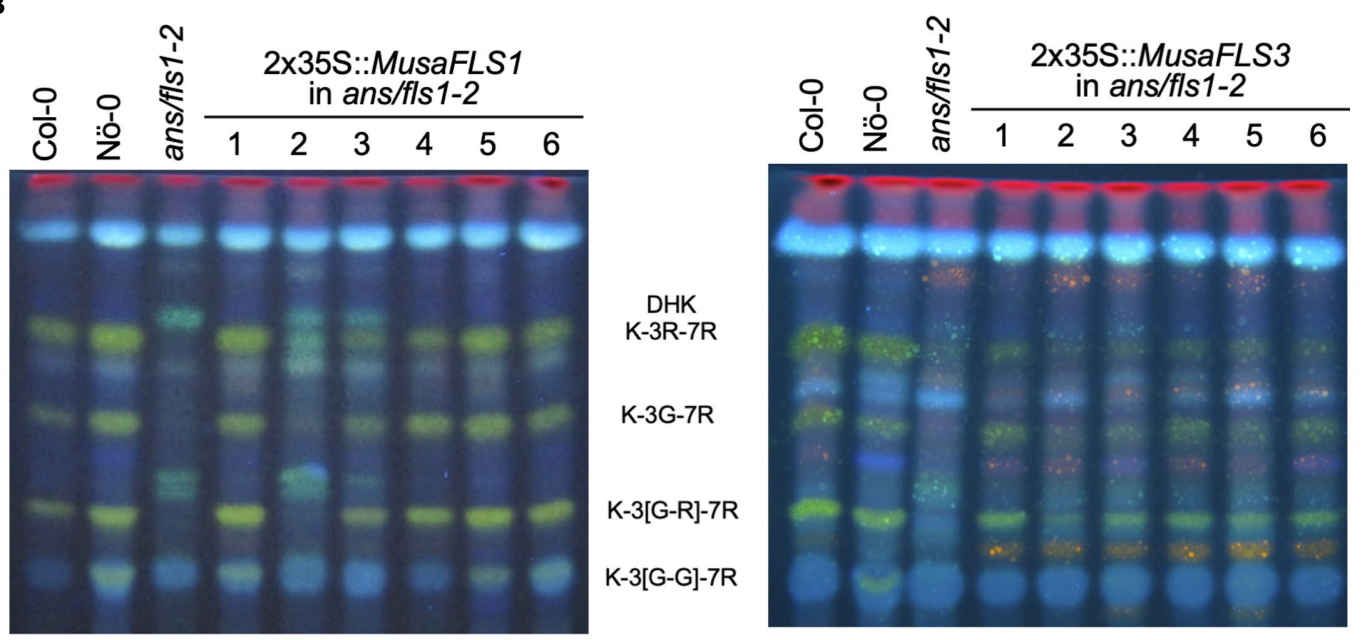

C
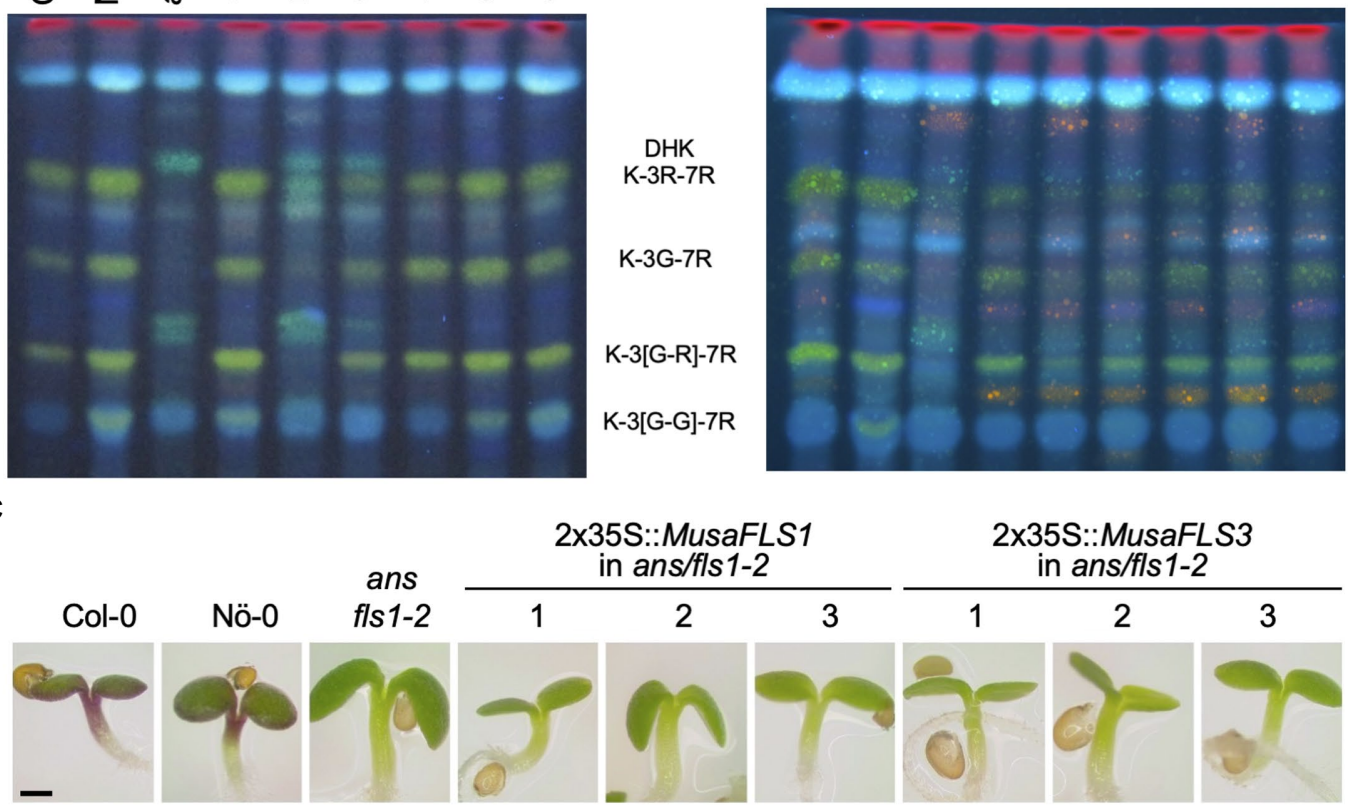

FIGURE 4 | MUsaFLS1 and MusaFLS3 are functional FLSs. (A) HPTLC analysis of a FLS bioconversion assays using extracts from E. coli expressing recombinant MusaFLS1 or MusaFLS3. The F3H substrate naringenin (N), the FLS substrate DHK and the product kaempferol (K) were used as standards. AtFLS1 served as a positive control and AtMYB12 was used as a negative control. (B) Flavonol glycoside accumulation in MusaFLS-complemented ans/fls1-2 seedlings analysed by HPTLC analysis. Col-0, Nö-0 (both wild type) and ans/fls1-2 were used as controls. Bright green spots belong to derivatives of kaempferol, orange spots are derivatives of quercetin and faint blue shows sinapate derivatives. Dark green and yellow spots indicate DHK and DHQ, respectively. G, glucose; K, kaempferol; Q, quercetin; and R, rhamnose. (C) Representative pictures of anthocyanin (red) accumulation in 6-day-old MusaFLS-complemented ans/fls1-2 seedlings growing on 4\% sucrose. The scale bar indicates $0.5 \mathrm{~mm}$.

\section{DISCUSSION}

In this study, we isolated several cDNAs of in silico annotated 2-ODD-type flavonoid biosynthesis enzyme coding genes from Musa and tested the in vivo functionality of the encoded proteins in E. coli and A. thaliana.

\section{MusaF3H1 and MusaF3H2}

In vivo E. coli bioconversion assays revealed that $M u s a \mathrm{~F} 3 \mathrm{H} 1$ and $M u s a \mathrm{~F} 3 \mathrm{H} 2$ can covert naringenin to DHK. Moreover,
MusaF3H1 and MusaF3H2 are able to complement the lossof-function phenotype of $A$. thaliana tt6-2 seedlings, showing in planta $\mathrm{F} 3 \mathrm{H}$ activity. Therefore, we conclude that $M u s a \mathrm{~F} 3 \mathrm{H} 1$ and $M u s a \mathrm{~F} 3 \mathrm{H} 2$ are functional $\mathrm{F} 3 \mathrm{Hs}$.

Previous studies annotated Ma02_g04650 (MusaF3H1) and Ma07_g17200 (MusaF3H2) as genes encoding F3Hs (Martin et al., 2016; Pandey et al., 2016; Pucker et al., 2020b). However, the automatic approach developed by Pucker et al. (2020b) also considered Ma07_g17200 as a candidate to encode a FNSI enzyme. For a Petroselinum crispum F3H protein, it has been found that a replacement of three amino 
A

B

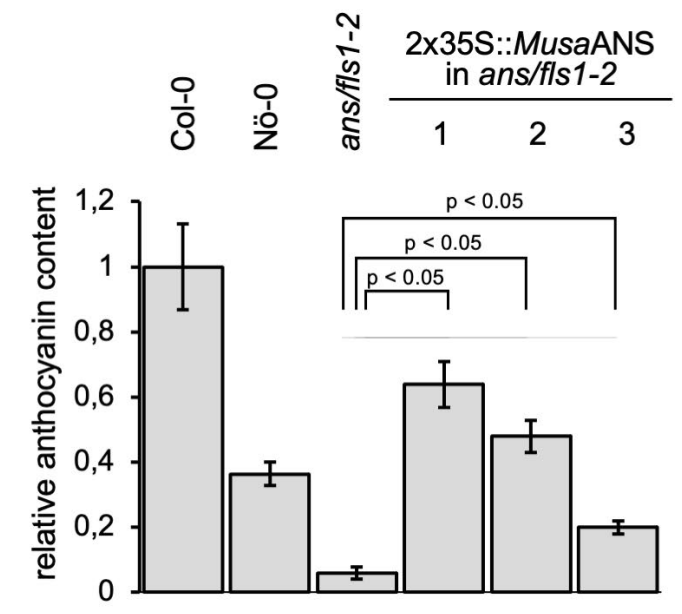

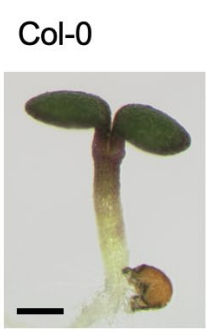

Nö-0

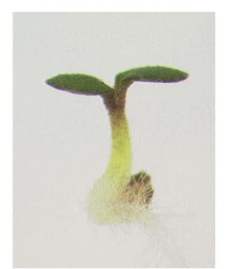

ans/fls1-2



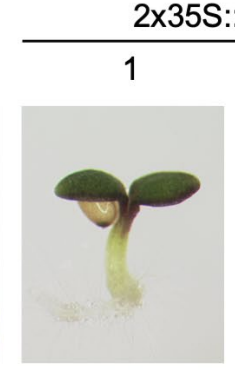

C

c

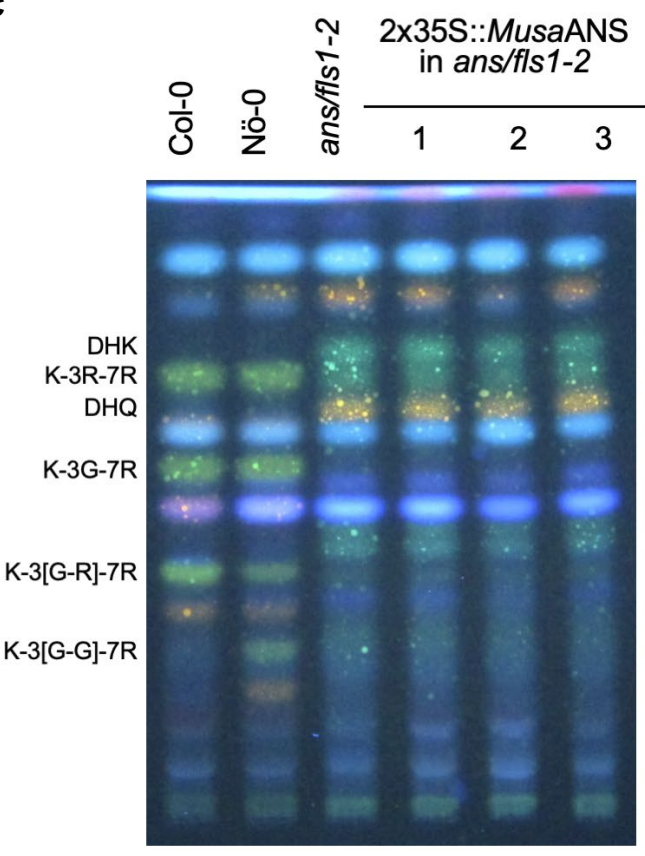

FIGURE 5 | MusaANS is a functional anthocyanin synthase. Analysis of ans/fls1-2 double mutant seedlings complemented with 2x35S-driven MusaANS demonstrate in planta ANS functionality of MusaANS by anthocyanin accumulation. The different numbers indicate individual transgenic lines. (A,B) Sucrose induced anthocyanin accumulation in 6-day-old Arabidopsis thaliana seedlings. (A) Representative pictures of seedlings (the scale bar indicates 0.5 mm) and (B) corresponding relative anthocyanin content. Error bars indicate the standard error for three independent measurements. (C) MusaANS does not show in planta FLS activity. Flavonol glycoside accumulation in MusaANS-complemented ans/fls 1-2 seedlings analysed by HPTLC analysis. Col-0, Nö-0 (both wild type) and ans/fls 1-2 were used as controls. Bright green spots belong to derivatives of kaempferol, orange spots are derivatives of quercetin and faint blue shows sinapate derivatives. Dark green and yellow spots indicate DHK and DHQ, respectively. G, glucose; K, kaempferol; Q, quercetin; and R, rhamnose.

acids was sufficient to cause FNSI side activity and seven amino acid exchanges almost lead to a complete change in enzyme activity towards FNSI functionality (Gebhardt et al., 2007). These findings underline the particular high similarity between FNSI and F3H. A closer inspection of the deduced peptide sequence of Ma07_g17200 gene revealed a lack of conservation of amino acid residues known to be relevant for FNSI function (i.e. T106M, T115I, I116V, F131I, E195D, I200V, V215L and R216K). Furthermore, Ma07_g17200 did not show FNSI activity in our bioconversion assay. Together with the confirmed $\mathrm{F} 3 \mathrm{H}$ activity, this indicates that the classification of Ma07_g17200 as a FNSI encoding gene was inaccurate. Even though Ma07_p17200 is no functional FNSI, flavone derivatives have been identified in Musa (Fu et al., 2018). In Gerbera and Glycine max, FNSII is responsible for the formation of flavones (Martens and Forkmann, 1999; Fliegmann et al., 2010). A candidate MusaFNSII, encoded by Ma08_g26160, has been identified (Pucker et al., 2020b) and is probably responsible for the accumulation of flavones in Musa. Since FNSII enzymes are NADPH-dependent cytochrome P450 monooxygenases (Jiang et al., 2016), the putative MusaFNSII (encoded by Ma08_g26160) was not studied in this work.

Our expression study that was based on published RNA-Seq data (Pucker et al., 2020a), revealed highest MusaF3H1 transcript abundance in early developmental stages of pulp and intermediate stages of peel development, while highest MusaF3H2 expression was found in adult leaves (Table 1). This is, at first glance, in contrast to the quantitative real-time PCR-derived expression data presented by Pandey et al. (2016), reporting high transcript 
levels of MusaF3H1 and MusaF3H2 in young leaves and bract. In this study, the authors also show increased MusaF3H1 expression in pseudostem, root and ripe pulp, compared to MusaF3H2. These discrepancies are probably due to the different growth conditions, germplasms/cultivars and sampling time points in the generation of the expression data and cannot be reasonably analysed further at this point. It should be noted, however, that MusaF3H2 expression in leaves of plantlets was found to be increased following treatment with the phytohormone methyl jasmonate (MJ), while MusaF3H1 expression was not (Pandey et al., 2016). As MJ is involved in various regulatory processes, including the response against biotic and abiotic stresses (summarised in Cheong and Choi, 2003), the MJ-dependent induction of $\mathrm{MusaF} 3 \mathrm{H} 2$ expression could imply that the MusaF3H2 enzyme plays a specialised role in the formation of flavonoids in response to stresses. Such stress response induction of $\mathrm{F} 3 \mathrm{H}$ encoding genes was previously shown for a $\mathrm{F} 3 \mathrm{H}$ gene from the dessert plant Reaumuria soongorica, which is induced by UV light (Liu et al., 2013) and two $\mathrm{F} 3 \mathrm{H}$ genes from Camellia sinensis, which are induced by UV light and by treatment with abscisic acid (ABA) or sucrose (Han et al., 2017). In addition, overexpression of $F 3 H$ from C. sinensis (Mahajan and Yadav, 2014) and from Lycium chinense (Song et al., 2016) in tobacco improved the tolerance to salt stress and fungal pathogens in the first case and to drought stress in the latter case. In conclusion, our results clearly show that $M u s a \mathrm{~F} 3 \mathrm{H} 1$ and $M u s a \mathrm{~F} 3 \mathrm{H} 2$ are functional $\mathrm{F} 3 \mathrm{Hs}$ and that $\mathrm{Musa \textrm {F } 3 \mathrm { H } 2}$ might play a role in stress response.

\section{MusaFLS1 and MusaFLS3}

MusaFLS1 was found to be able to convert DHK and DHQ to the corresponding flavonol in the E. coli bioconversion assays. This ability was validated in planta by successful complementation of the flavonol deficiency of $A$. thaliana ans/fls1-2 double mutant seedlings, while the anthocyanin deficiency phenotype was not restored. These observations could hint to an exclusive FLS activity of MusaFLS1. FLS and DFR, the first enzymes of the flavonol and anthocyanin branches of flavonoid biosynthesis, compete for dihydroflavonol substrates (Figure 1). This is demonstrated by several $A$. thaliana $\mathrm{fls} 1$ single mutants which accumulate higher levels of anthocyanin pigments (Owens et al., 2008; Stracke et al., 2009). Falcone Ferreyra et al. (2010) found that overexpression of ZmFLS1 in an A. thaliana fls1 mutant decreases the accumulation of anthocyanins to wild-type level, again indicating that the anthocyanin and flavonol branches of flavonoid biosynthesis can compete for dihydroflavonol substrates in the same cell or tissue. Accordingly, the overlapping substrate usage of FLS and ANS is difficult to analyse in a fls 1 single mutant. Here, the use of an $A$. thaliana ans/fls1-2 double mutant is a simple way to analyse the FLS and a possible ANS side activity of FLSs and vice versa in planta. To analyse further side activities, a $f 3 h \mathrm{fls}$ ans mutant would be beneficial. A. thaliana ans/fls1-2 plants expressing MusaFLS3 showed the accumulation of several kaempferol derivatives. In contrast, plants complemented with MusaFLS1 also accumulate quercetin derivatives. This is most likely an effect of different environmental conditions in plant growth. One set of plants was grown in the greenhouse (MusaFLS1), another set was grown in a growth chamber (MusaFLS3). In the plants used for MusaFLS3 experiments, these conditions promoted the accumulation of DHQ, while the other growth conditions did not in the plants used for MusaFLS1 experiments. Accordingly, DHQ was probably not available as a substrate for MusaFLS1 and could not be converted to quercetin. A possible explanation for the varying DHQ amounts could be the light-induced expression of flavonoid 3'hydroxylase (F3'H), which converts DHK to DHQ. The expression of F3' $H$ in cultured $A$. thaliana cells can be induced by UV light (Schoenbohm et al., 2000) and the activity of the F3' $H$ promoter from Vitis vinifera is increased under light exposure (Sun et al., 2015). Consequently, a different light quality or higher light intensity could be responsible for increased F3' $H$ expression, causing the accumulation of DHQ in the plants grown in the growth chamber. While MusaFLS3 was able to complement the flavonol deficiency in the ans/fls $1-2$ mutant, it did not lead to an accumulation of anthocyanins. In contrast to the in planta results, MusaFLS3 did not convert DHK or DHQ to the corresponding flavonol in the in vivo bioconversion assays, even though the protein was successfully expressed in the E. coli culture. We used the bioconversion assays as a simple and versatile tool to analyse enzymatic activities. Nevertheless, heterologous expression of eukaryotic proteins in $E$. coli is an artificial system. It can cause a divergent pattern of post-translational modifications or production of high amounts of protein in inclusion bodies, leading to inactive protein (Sahdev et al., 2008). Preuss et al. (2009) reported that AtFLS3 did not show FLS activity in E. coli, but did convert dihydroflavonols to the corresponding flavonols upon expression in yeast, indicating that the stability of AtFLS3 was improved under the latter assay conditions. The approach carried out in this work might have similar limitations. Further limitations can be caused by different substrate preferences, as reported for OsFLS (Park et al., 2019). Despite the lack of FLS activity in the bioconversion assay, MusaFLS3 showed F3H activity. Nevertheless, the in planta complementation of the flavonol deficiency in the A. thaliana ans/fls1-2 mutant and the lacking complementation of the anthocyanin deficiency phenotype in the ans/fls1-2 mutant indicate that MusaFLS3 is a bifunctional enzyme with FLS and $\mathrm{F} 3 \mathrm{H}$ activity, which does not exhibit significant ANS activity.

MusaFLS1 transcript abundance was found to be high in adult leaves and low in roots. MusaFLS3 and MusaFLS4 revealed opposed transcript levels. Such divergent expression patterns have previously been observed for FLS1 and FLS2 from Freesia hybrida and FLS1 and FLS2 from Cyclamen purpurascens (Akita et al., 2018; Shan et al., 2020). These opposed expression patterns could point to differential activity in distinct organs. Furthermore, the tandemly arranged MusaFLS3 and MusaFLS4 genes show very similar expression patterns (Table 1), possibly indicating functional redundancy. While we could not find expression of MusaFLS2 in our expression analyses, Pandey 
et al. (2016) observed MusaFLS2 expression in several organs (including bract, pseudostem and root), as well as in different developmental stages of peel and pulp. Again, these results show that different cultivars, growth conditions, sampling time points and analysed organs can have a strong influence on the resulting data. Accordingly, data from different studies should be evaluated carefully. MusaFLS1 and MusaFLS2 expression in leaves of plantlets does not significantly increase after MJ treatment (Pandey et al., 2016). However, UV radiation induces the expression of FLS from Z. mays (Falcone Ferreyra et al., 2010) and M. domestica (Henry-Kirk et al., 2018) and the relative expression of FLS from Triticum aestivum increases during drought stress (Ma et al., 2014). Together with the knowledge that flavonols act as antioxidants (Wang et al., 2006), an involvement of MusaFLSs in stress response seems feasible and should be further analysed under a broader range of conditions. In summary, our results indicate that MusaFLS1 and MusaFLS3 are functional FLS enzymes and hint at possible differential organ-specific activities of MusaFLS1 and MusaFLS3/MusaFLS4.

\section{MusaANS}

A. thaliana ans/fls1-2 seedlings expressing MusaANS show a strong, red pigmentation, revealing that MusaANS can complement the anthocyanin deficiency caused by mutation of AtANS. However, the seedlings did not display flavonol derivatives in HPTLC analyses, which have been reported to detect flavonol glycosides at levels of $50 \mathrm{pMol}$ (Stracke et al., 2010). These results indicate that MusaANS is a functional ANS but does not have FLS activity. The RNA-Seq data-derived expression profiles revealed high MusaANS transcript abundance in pulp and peel. Additionally, MusaANS expression has been reported to be high in bract tissue (Pandey et al., 2016), the specialised leaves surrounding the flowers and usually coloured red or purple due to anthocyanin accumulation (Pazmiño-Durána et al., 2001). This spatial correlation of MusaANS transcripts and anthocyanin metabolites in bract tissue supports the proposed biological functionality of this enzyme, catalysing the conversion of leucoanthocyanidins to anthocyanidins in Musa. MusaANS expression increases $24 \mathrm{~h}$ after MJ treatment and decreases following dark treatment (Pandey et al., 2016). As supposed for MusaF3H2, the increased expression of MusaANS after MJ treatment could imply an involvement in the formation of anthocyanins as a consequence of stress response. ABA, salicylic acid (SA), UV-B and cold treatments have been shown to enhance ANS transcript abundance in G. biloba (Xu et al., 2008) and overexpression of OsANS raises the antioxidant potential in $O$. sativa (Reddy et al., 2007). The accumulation of anthocyanins has often been shown to be induced by (UV-) light (Takahashi et al., 1991; Stapleton and Walbot, 1994; Meng et al., 2004). We therefore assume that also MusaANS could be involved in such stress response.

Very recently, a R2R3-MYB-type transcription factor (MaMYB4) has been identified as a negative regulator of anthocyanin biosynthesis in Musa acting as repressor on the MusaANS promoter (Deng et al., 2021). These results give a first insight into the transcriptional regulation of MusaANS expression and confirm the role of MusaANS in the anthocyanin biosynthesis in Musa. Taking all available evidence into account, MusaANS encodes a functional ANS with a possible involvement in the plants' stress response.

To deepen the knowledge about 2-ODDs and other enzymes involved in Musa flavonoid biosynthesis, it would be beneficial to acquire even more spatially and timely highly resolved transcriptome and in particular metabolite data. This data could serve as a starting point for the analysis of organ-, stress- or development-specific enzyme activities and functions, as well as possible substrate preferences. It could also be used to elucidate the regulatory network of Musa flavonoid biosynthesis. Furthermore, knowledge about the influence of different stresses (e.g. pathogens, light and temperature) on specific transcriptomes and metabolomes of the Musa plant could help to widen the knowledge of flavonoid biosynthesis and particular the functionality of the Musa 2-ODD enzymes. This could also lead to the detection of possible restricted side activities or overlapping functionalities as described for 2-OODs in some other plant species (Falcone Ferreyra et al., 2010; Park et al., 2019) and to further decode the cause of these multifunctionalities in 2-ODDs.

To conclude, in this study, the functionality of five 2-ODDs involved in flavonoid biosynthesis in Musa was demonstrated in vivo in bacterial cells and in planta. Knowledge gained about the structural genes MusaF3H1, MusaF3H2, MusaFLS1, MusaFLS3 and MusaANS in a major crop plant provides a basis for further research towards engineered, increased flavonoid production in banana, which could contribute to the fruits' antioxidant activity and nutritional value, and possibly even enhanced the plants' defence against Foc-TR4.

\section{DATA AVAILABILITY STATEMENT}

The original contributions presented in the study are included in the article/Supplementary Material, and further inquiries can be directed to the corresponding author.

\section{AUTHOR CONTRIBUTIONS}

$\mathrm{MB}$ and RS planned the experiments. $\mathrm{MB}$ and $\mathrm{CA}$ performed the experiments and analysed the data. $\mathrm{MB}$ and $\mathrm{SM}$ interpreted the TLC data. RS and BW supervised the project and revised the manuscript. $\mathrm{MB}$ wrote the initial draft. All authors read and approved the final manuscript version.

\section{FUNDING}

This work was supported by the basic funding of the chair of Genetics and Genomics of Plants provided by the Bielefeld 
University/Faculty of Biology and the Open Access Publication Fund of Bielefeld University.

\section{ACKNOWLEDGMENTS}

We are grateful to Melanie Kuhlmann for her excellent assistance in the laboratory and to Andrea Voigt for her competent help in the greenhouse. We thank Anika Beckers who contributed to creation of constructs with MusaFLS cDNAs and Prisca Viehöver for sequencing. In addition,

\section{REFERENCES}

Akita, Y., Kitamura, S., Mikami, R., and Ishizaka, H. (2018). Identification of functional flavonol synthase genes from fragrant wild cyclamen (Cyclamen purpurascens). J. Plant Biochem. Biotechnol. 27, 147-155. doi: 10.1007/s13562-017-0423-9

Andersen, Ø. M., and Jordheim, M. (2010). "Anthocyanins," in Encyclopedia of Life Sciences (Chichester: John Wiley \& Sons, Ltd).

Appelhagen, I., Thiedig, K., Nordholt, N., Schmidt, N., Huep, G., Sagasser, M., et al. (2014). Update on transparent testa mutants from Arabidopsis thaliana: characterisation of new alleles from an isogenic collection. Planta 240, 955-970. doi: 10.1007/s00425-014-2088-0

Arias, P., Dankers, C., Liu, P., and Pilkauskas, P. (2003). The World Banana Economy, 1985-2002. Rome, Italy: Food and Agricultural Organization (FAO).

Asif, M. H., Dhawan, P., and Nath, P. (2000). A simple procedure for the isolation of high quality RNA from ripening banana fruit. Plant Mol. Biol. Report. 18, 109-115. doi: 10.1007/BF02824018

Bednar, R. A., and Hadcock, J. R. (1988). Purification and characterizationo of chalcone isomerase from soybeans. J. Biol. Chem. 263, 9582-9588. doi: 10.1016/S0021-9258(19)81556-9

Britsch, L. (1990). Purification and characterization of flavone synthase I, a 2-oxoglutarate-dependent desaturase. Arch. Biochem. Biophys. 282, 152-160. doi: 10.1016/0003-9861(90)90099-K

Brown, J. W., Walker, J. F., and Smith, S. A. (2017). Phyx: phylogenetic tools for unix. Bioinformatics 33, 1886-1888. doi: 10.1093/bioinformatics/btx063

Cheng, A. X., Han, X. J., Wu, Y. F., and Lou, H. X. (2014). The function and catalysis of 2-oxoglutarate-dependent oxygenases involved in plant flavonoid biosynthesis. Int. J. Mol. Sci. 15, 1080-1095. doi: 10.3390/ijms15011080

Cheong, J.- J., and Choi, Y. D. (2003). Methyl jasmonate as a vital substance in plants. Trends Genet. 19, 409-413. doi: 10.1016/S0168-9525(03)00138-0

Clough, S. J., and Bent, A. F. (1998). Floral dip: a simplified method for agrobacterium-mediated transformation of Arabidopsis thaliana. Plant J. 16, 735-743. doi: 10.1046/j.1365-313x.1998.00343.x

Dauer, A., Hensel, A., Lhoste, E., Knasmüller, S., and Mersch-Sundermann, V. (2003). Genotoxic and antigenotoxic effects of catechin and tannins from the bark of Hamamelis virginiana L. in metabolically competent, human hepatoma cells (hep G2) using single cell gel electrophoresis. Phytochemistry 63, 199-207. doi: 10.1016/S0031-9422(03)00104-3

Deng, G. M., Zhang, S., Yang, Q. S., Gao, H. J., Sheng, O., Bi, F. C., et al. (2021). MaMYB4, an R2R3-MYB repressor transcription factor, negatively regulates the biosynthesis of anthocyanin in banana. Front. Plant Sci. 11:600704. doi: $10.3389 /$ fpls.2020.600704

DHont, A., Denoeud, F., Aury, J. M., Baurens, F. C., Carreel, F., Garsmeur, O., et al. (2012). The banana (Musa acuminata) genome and the evolution of monocotyledonous plants. Nature 488, 213-217. doi: 10.1038/nature11241

Falcone Ferreyra, M. L., Rius, S. P., and Casati, P. (2012). Flavonoids: biosynthesis, biological functions, and biotechnological applications. Front. Plant Sci. 3:222. doi: $10.3389 /$ fpls.2012.00222

Falcone Ferreyra, M. L., Rius, S., Emiliani, J., Pourcel, L., Feller, A., Morohashi, K., et al. (2010). Cloning and characterization of a UV-B-inducible maize flavonol synthase. Plant J. 62, 77-91. doi: 10.1111/j.1365-313X.2010.04133.x

FAO (2019). The State of Food and Agriculture 2019. Rome, Italy: Food and Agricultural Organization (FAO). we thank Thomas Baier for his support with the SDS-PAGE and Ashutosh Pandey for providing us with the banana cDNAs. We acknowledge support for the publication costs by the Open Access Publication Fund of Bielefeld University.

\section{SUPPLEMENTARY MATERIAL}

The Supplementary Material for this article can be found online at https://www.frontiersin.org/articles/10.3389/fpls.2021.701780/ full\#supplementary-material

Fliegmann, J., Furtwangler, K., Malterer, G., Cantarello, C., Schuler, G., Ebel, J., et al. (2010). Flavone synthase II (CYP93B16) from soybean (Glycine max L.). Phytochemistry 71, 508-514. doi: 10.1016/j.phytochem.2010.01.007

Forkmann, G., de Vlaming, P., Spribille, R., Wiering, H., and Schram, W. (1986). Genetic and biochemical studies on the conversion of dihydroflavonols to flavonols in flowers of Petunia hybrida. Zeitschrift für Naturforschung $C$ 41, 179-186. doi: 10.1515/znc-1986-1-227

Forkmann, G., Heller, W., and Grisebach, H. (1980). Anthocyanin biosynthesis in flowers of Matthiola incana flavanone 3-and flavonoid 3'-hydroxylases. Zeitschrift für Naturforschung C 35, 691-695. doi: 10.1515/znc-1980-9-1004

Forster, M., Rodríguez, E. R., Martín, J. D., and Romero, C. D. (2003). Distribution of nutrients in edible banana pulp. Food Technol. Biotechnol. 41, 167-171.

Fu, X., Cheng, S., Liao, Y., Huang, B., Du, B., Zeng, W., et al. (2018). Comparative analysis of pigments in red and yellow banana fruit. Food Chem. 239, 1009-1018. doi: 10.1016/j.foodchem.2017.07.046

Gebhardt, Y. H., Witte, S., Steuber, H., Matern, U., and Martens, S. (2007). Evolution of flavone synthase I from parsley flavanone 3beta-hydroxylase by site-directed mutagenesis. Plant Physiol. 144, 1442-1454. doi: 10.1104/pp.107.098392

Goufo, P., and Trindade, H. (2014). Rice antioxidants: phenolic acids, flavonoids, anthocyanins, proanthocyanidins, tocopherols, tocotrienols, gamma-oryzanol, and phytic acid. Food Sci. Nutr. 2, 75-104. doi: 10.1002/fsn3.86

Gronquist, M., Bezzerides, A., Attygalle, A., Meinwald, J., Eisner, M., and Eisner, T. (2001). Attractive and defensive functions of the ultraviolet pigments of a flower (Hypericum calycinum). Proc. Natl. Acad. Sci. U. S. A. 98, 13745-13750. doi: 10.1073/pnas.231471698

Grotewold, E. (2006). The Science of Flavonoids. Columbus: The Ohio State University.

Hahlbrock, K., and Scheel, D. (1989). Physiology and molecular biology of phenylpropanoid metabolism. Annu. Rev. Plant Physiol. Plant Mol. Biol. 40, 347-369. doi: 10.1146/annurev.pp.40.060189.002023

Han, Y., Huang, K., Liu, Y., Jiao, T., Ma, G., Qian, Y., et al. (2017). Functional analysis of two flavanone-3-hydroxylase genes from Camellia sinensis: a critical role in flavonoid accumulation. Genes 8:300. doi: 10.3390/genes8110300

Heller, W., Forkmann, G., Britsch, L., and Grisebach, H. (1985). Enzymatic reduction of (+)-dihydroflavonols to flavan-3,4-cis-diols with flower extracts from Matthiola incana and its role in anthocyanin biosynthesis. Planta 165 284-287. doi: 10.1007/BF00395052

Henry-Kirk, R. A., Plunkett, B., Hall, M., McGhie, T., Allan, A. C., Wargent, J. J., et al. (2018). Solar UV light regulates flavonoid metabolism in apple (Malus $\mathrm{x}$ domestica). Plant Cell Environ. 41, 675-688. doi: 10.1111/pce.13125

Holton, T. A., Brugliera, F., and Tanaka, Y. (1993). Cloning and expression of flavonol synthase from Petunia hybrida. Plant J. 4, 1003-1010. doi: 10.1046/j. 1365-313X.1993.04061003.x

Ishikura, N., and Yoshitama, K. (1984). Anthocyanin-Flavonol co-pigmentation in blue seed coats of Ophiopogon jaburan. J. Plant Physiol. 115, 171-175. doi: 10.1016/S0176-1617(84)80064-4

Jaakkola, P., Mole, D. R., Tian, Y. M., Wilson, M. I., Gielbert, J., Gaskell, S. J., et al. (2001). Targeting of HIF-alpha to the von Hippel-Lindau ubiquitylation complex by O2-regulated prolyl hydroxylation. Science 292, 468-472. doi: $10.1126 /$ science. 1059796

Jakoby, M., Wang, H. Y., Reidt, W., Weisshaar, B., and Bauer, P. (2004). FRU (BHLH029) is required for induction of iron mobilization genes in Arabidopsis thaliana. FEBS Lett. 577, 528-534. doi: 10.1016/j.febslet.2004.10.062 
Jiang, N., Doseff, A. I., and Grotewold, E. (2016). Flavones: from biosynthesis to health benefits. Plan. Theory 5:27. doi: 10.3390/plants5020027

Kandil, F. E., Smith, M. A., Rogers, R. B., Pepin, M. F., Song, L. L., Pezzuto, J. M., et al. (2002). Composition of a chemopreventive proanthocyanidin-rich fraction from cranberry fruits responsible for the inhibition of 12-O-tetradecanoyl phorbol-13-acetate (TPA)-induced ornithine decarboxylase (ODC) activity. J. Agric. Food Chem. 50, 1063-1069. doi: 10.1021/jf011136z

Katoh, K., and Standley, D. M. (2013). MAFFT multiple sequence alignment software version 7: improvements in performance and usability. Mol. Biol. Evol. 30, 772-780. doi: 10.1093/molbev/mst010

Kawai, Y., Ono, E., and Mizutani, M. (2014). Evolution and diversity of the 2-oxoglutarate-dependent dioxygenase superfamily in plants. Plant J. 78, 328-343. doi: $10.1111 /$ tpj.12479

Koncz, C., and Schell, J. (1986). The promoter of TL-DNA gene 5 controls the tissue-specific expression of chimaeric genes carried by a novel type of agrobacterium binary vector. Mol. Genet. Genomics 204, 383-396. doi: 10.1007/ BF00331014

Kreuzaler, F., and Hahlbrock, K. (1972). Enzymatic synthesis of aromatic compounds in higher plants: formation of naringenin (5,7,4'-trihydroxyflavanone) from p-coumaroyl coenzyme A and malonyl coenzyme A. FEBS Lett. 28, 69-72. doi: 10.1016/0014-5793(72)80679-3

Le Roy, J., Huss, B., Creach, A., Hawkins, S., and Neutelings, G. (2016). Glycosylation is a major regulator of phenylpropanoid availability and biological activity in plants. Front. Plant Sci. 7:735. doi: 10.3389/ fpls.2016.00735

Lepiniec, L., Debeaujon, I., Routaboul, J. M., Baudry, A., Pourcel, L., Nesi, N., et al. (2006). Genetics and biochemistry of seed flavonoids. Annu. Rev. Plant Biol. 57, 405-430. doi: 10.1146/annurev.arplant.57.032905.105252

Letunic, I., and Bork, P. (2019). Interactive tree Of life (iTOL) v4: recent updates and new developments. Nucleic Acids Res. 47, W256-W259. doi: 10.1093/nar/gkz239

Li, J., Ou-Lee, T. M., Raba, R., Amundson, R. G., and Last, R. L. (1993). Arabidopsis flavonoid mutants are hypersensitive to UV-B irradiation. Plant Cell 5, 171-179. doi: 10.2307/3869583

Liu, M., Li, X., Liu, Y., and Cao, B. (2013). Regulation of flavanone 3-hydroxylase gene involved in the flavonoid biosynthesis pathway in response to UV-B radiation and drought stress in the desert plant, Reaumuria soongorica. Plant Physiol. Biochem. 73, 161-167. doi: 10.1016/j.plaphy.2013.09.016

Lu, P., Yu, S., Zhu, N., Chen, Y. R., Zhou, B., Pan, Y., et al. (2018). Genome encode analyses reveal the basis of convergent evolution of fleshy fruit ripening. Nat. Plants 4, 784-791. doi: 10.1038/s41477-018-0249-z

Luo, P., Ning, G., Wang, Z., Shen, Y., Jin, H., Li, P., et al. (2016). Disequilibrium of flavonol synthase and dihydroflavonol-4-reductase expression associated tightly to white vs. red color flower formation in plants. Front. Plant Sci. 6:1257. doi: 10.3389/fpls.2015.01257

Ma, D. Y., Sun, D. X., Wang, C. Y., Li, Y. G., and Guo, T. C. (2014). Expression of flavonoid biosynthesis genes and accumulation of flavonoid in wheat leaves in response to drought stress. Plant Physiol. Biochem. 80, 60-66. doi: 10.1016/j.plaphy.2014.03.024

Mahajan, M., and Yadav, S. K. (2014). Overexpression of a tea flavanone 3-hydroxylase gene confers tolerance to salt stress and Alternaria solani in transgenic tobacco. Plant Mol. Biol. 85, 551-573. doi: 10.1007/s11103-014-0203-Z

Mann, H. B., and Whitney, D. R. (1947). On a test of Whether one of two random variables is stochastically larger than the other. Ann. Math. Stat. 18, 50-60. doi: 10.1214/aoms/1177730491

Martens, S., and Forkmann, G. (1999). Cloning and expression of flavone synthase II from Gerbera hybrids. Plant J. 20, 611-618. doi: 10.1046/j.1365-313X. 1999.00636.x

Martens, S., Forkmann, G., Britsch, L., Wellmann, F., Matern, U., and Lukacin, R. (2003). Divergent evolution of flavonoid 2-oxoglutaratedependent dioxygenases in parsley. FEBS Lett. 544, 93-98. doi: 10.1016/ S0014-5793(03)00479-4

Martin, G., Baurens, F. C., Droc, G., Rouard, M., Cenci, A., Kilian, A., et al. (2016). Improvement of the banana "Musa acuminata," reference sequence using NGS data and semi-automated bioinformatics methods. BMC Genomics 17:243. doi: 10.1186/s12864-016-2579-4

Mehrtens, F., Kranz, H., Bednarek, P., and Weisshaar, B. (2005). The Arabidopsis transcription factor MYB12 is a flavonol-specific regulator of phenylpropanoid biosynthesis. Plant Physiol. 138, 1083-1096. doi: 10.1104/pp.104.058032
Meng, X., Xing, T., and Wang, X. (2004). The role of light in the regulation of anthocyanin accumulation in Gerbera hybrida. Plant Growth Reg. 44, 243-250. doi: 10.1007/s10725-004-4454-6

Mo, Y., Nagel, C., and Taylor, L. P. (1992). Biochemical complementation of chalcone synthase mutants defines a role for flavonols in functional pollen. Proc. Natl. Acad. Sci. U. S. A. 89, 7213-7217. doi: 10.1073/pnas.89.15.7213

Owens, D. K., Alerding, A. B., Crosby, K. C., Bandara, A. B., Westwood, J. H., and Winkel, B. S. (2008). Functional analysis of a predicted flavonol synthase gene family in Arabidopsis. Plant Physiol. 147, 1046-1061. doi: 10.1104/ pp.108.117457

Pandey, A., Alok, A., Lakhwani, D., Singh, J., Asif, M. H., and Trivedi, P. K. (2016). Genome-wide Expression analysis and metabolite profiling elucidate transcriptional regulation of flavonoid biosynthesis and modulation under abiotic stresses in banana. Sci. Rep. 6:31361. doi: 10.1038/srep31361

Park, S., Kim, D. H., Park, B. R., Lee, J. Y., and Lim, S. H. (2019). Molecular and functional characterization of Oryza sativa Flavonol synthase (OsFLS), a Bifunctional dioxygenase. J. Agric. Food Chem. 67, 7399-7409. doi: 10.1021/ acs.jafc.9b02142

Pazmiño-Durána, E. A., Giustib, M. M., Wrolstad, R. E., and Glóriaa, M. B. A. (2001). Anthocyanins from banana bracts (Musa X paradisiaca) as potential food colorants. Food Chem. 73, 327-332. doi: 10.1016/ S0308-8146(00)00305-8

Perez-Vizcaino, F., and Duarte, J. (2010). Flavonols and cardiovascular disease. Mol. Asp. Med. 31, 478-494. doi: 10.1016/j.mam.2010.09.002

Prescott, A. G., and John, P. (1996). DIOXYGENASES: molecular structure and role in plant metabolism. Annu. Rev. Plant Physiol. Plant Mol. Biol. 47, 245-271. doi: 10.1146/annurev.arplant.47.1.245

Preuss, A., Stracke, R., Weisshaar, B., Hillebrecht, A., Matern, U., and Martens, S. (2009). Arabidopsis thaliana expresses a second functional flavonol synthase. FEBS Lett. 583, 1981-1986. doi: 10.1016/j.febslet.2009.05.006

Price, M. N., Dehal, P. S., and Arkin, A. P. (2010). FastTree 2-approximately maximum-likelihood trees for large alignments. PLoS One 5:e9490. doi: 10.1371/journal.pone.0009490

Pucker, B., Pandey, A., Weisshaar, B., and Stracke, R. (2020a). The R2R3-MYB gene family in banana (Musa acuminata): genome-wide identification, classification and expression patterns. PLoS One 15:e0239275. doi: 10.1371/ journal.pone.0239275

Pucker, B., Reiher, F., and Schilbert, H. M. (2020b). Automatic identification of players in the flavonoid biosynthesis with application on the biomedicinal plant Croton tiglium. Plan. Theory 9:1103. doi: 10.3390/plants9091103

Reddy, A. M., Reddy, V. S., Scheffler, B. E., Wienand, U., and Reddy, A. R. (2007). Novel transgenic rice overexpressing anthocyanidin synthase accumulates a mixture of flavonoids leading to an increased antioxidant potential. Metab. Eng. 9, 95-111. doi: 10.1016/j.ymben.2006.09.003

Sahdev, S., Khattar, S. K., and Saini, K. S. (2008). Production of active eukaryotic proteins through bacterial expression systems: a review of the existing biotechnology strategies. Mol. Cell. Biochem. 307, 249-264. doi: 10.1007/ s11010-007-9603-6

Saito, K., Kobayashi, M., Gong, Z. Z., Tanaka, Y., and Yamazaki, M. (1999). Direct evidence for anthocyanidin synthase as a 2-oxoglutarate-dependent oxygenase: molecular cloning and functional expression of cDNA from a red forma of Perilla frutescens. Plant J. 17, 181-189. doi: 10.1046/j.1365-313X.1999.00365.X

Sanger, F., Nicklen, S., and Coulson, A. R. (1977). DNA sequencing with chainterminating inhibitors. Proc. Natl. Acad. Sci. U. S. A. 74, 5463-5467. doi: 10.1073/pnas.74.12.5463

Schoenbohm, C., Martens, S., Eder, C., Forkmann, G., and Weisshaar, B. (2000). Identification of the Arabidopsis thaliana flavonoid 3'-hydroxylase gene and functional expression of the encoded P450 enzyme. Biol. Chem. 381, 749-753. doi: 10.1515/BC.2000.095

Shan, X., Li, Y., Yang, S., Yang, Z., Qiu, M., Gao, R., et al. (2020). The spatiotemporal biosynthesis of floral flavonols is controlled by differential phylogenetic MYB regulators in Freesia hybrida. New Phytol. 228, 1864-1879. doi: 10.1111/ nph. 16818

Sheahan, J. J., and Rechnitz, G. A. (1992). Flavonoid-specific staining of Arabidopsis thaliana. BioTechniques 13, 880-883.

Singh, B., Singh, J. P., Kaur, A., and Singh, N. (2016). Bioactive compounds in banana and their associated health benefits - A review. Food Chem. 206, 1-11. doi: 10.1016/j.foodchem.2016.03.033 
Song, X., Diao, J., Ji, J., Wang, G., Guan, C., Jin, C., et al. (2016). Molecular cloning and identification of a flavanone 3-hydroxylase gene from Lycium chinense, and its overexpression enhances drought stress in tobacco. Plant Physiol. Biochem. 98, 89-100. doi: 10.1016/j.plaphy.2015.11.011

Stapleton, A. E., and Walbot, V. (1994). Flavonoids can protect maize DNA from the induction of ultraviolet radiation damage. Plant Physiol. 105, 881-889. doi: 10.1104/pp.105.3.881

Stracke, R., De Vos, R. C. H., Bartelniewoehner, L., Ishihara, H., Sagasser, M., Martens, S., et al. (2009). Metabolomic and genetic analyses of flavonol synthesis in Arabidopsis thaliana support the in vivo involvement of leucoanthocyanidin dioxygenase. Planta 229, 427-445. doi: 10.1007/ s00425-008-0841-y

Stracke, R., Ishihara, H., Huep, G., Barsch, A., Mehrtens, F., Niehaus, K., et al. (2007). Differential regulation of closely related R2R3-MYB transcription factors controls flavonol accumulation in different parts of the Arabidopsis thaliana seedling. Plant J. 50, 660-677. doi: 10.1111/j.1365-313X.2007.03078.x

Stracke, R., Jahns, O., Keck, M., Tohge, T., Niehaus, K., Fernie, A. R., et al. (2010). Analysis of PRODUCTION OF FLAVONOL GLYCOSIDES-dependent flavonol glycoside accumulation in Arabidopsis thaliana plants reveals MYB11-, MYB12- and MYB111-independent flavonol glycoside accumulation. New Phytol. 188, 985-1000. doi: 10.1111/j.1469-8137.2010.03421.x

Stracke, R., Turgut-Kara, N., and Weisshaar, B. (2017). The AtMYB12 activation domain maps to a short C-terminal region of the transcription factor. Z. Naturforsch. C. J. Biosci. 72, 251-257. doi: 10.1515/znc-2016-0221

Sun, R. Z., Pan, Q. H., Duan, C. Q., and Wang, J. (2015). Light response and potential interacting proteins of a grape flavonoid 3'-hydroxylase gene promoter. Plant Physiol. Biochem. 97, 70-81. doi: 10.1016/j.plaphy. 2015.09.016

Sun, J., Zhang, J., Fang, H., Peng, L., Wei, S., Li, C., et al. (2019). Comparative transcriptome analysis reveals resistance-related genes and pathways in Musa acuminata banana 'Guijiao 9' in response to Fusarium wilt. Plant Physiol. Biochem. 141, 83-94. doi: 10.1016/j.plaphy.2019.05.022

Takahashi, A., Takeda, K., and Ohnishi, T. (1991). Light-induced anthocyanin reduces the extent of damage to DNA in UV-irradiated Centaurea cyanus cells in culture. Plant Cell Physiol. 32, 541-547. doi: 10.1093/oxfordjournals. pcp.a078113

Tanaka, Y., Sasaki, N., and Ohmiya, A. (2008). Biosynthesis of plant pigments: anthocyanins, betalains and carotenoids. Plant J. 54, 733-749. doi: 10.1111/j. 1365-313X.2008.03447.x

Tohge, T., de Souza, L. P., and Fernie, A. R. (2017). Current understanding of the pathways of flavonoid biosynthesis in model and crop plants. J. Exp. Bot. 68, 4013-4028. doi: 10.1093/jxb/erx177
Trewick, S. C., Henshaw, T. F., Hausinger, R. P., Lindahl, T., and Sedgwick, B. (2002). Oxidative demethylation by Escherichia coli AlkB directly reverts DNA base damage. Nature 419, 174-178. doi: 10.1038/nature00908

Wall, M. M. (2006). Ascorbic acid, vitamin A, and mineral composition of banana (Musa sp.) and papaya (Carica papaya) cultivars grown in Hawaii. J. Food Compost. Anal. 19, 434-445. doi: 10.1016/j.jfca.2006.01.002

Wang, L., Tu, Y.- C., Lian, T.- W., Hung, J.- T., Yen, J.- H., and Wu, M.- J. (2006). Distinctive antioxidant and antiinflammatory effects of flavonols. J. Agric. Food Chem. 54, 9798-9804. doi: 10.1021/jf0620719

Wen, W., Alseekh, S., and Fernie, A. R. (2020). Conservation and diversification of flavonoid metabolism in the plant kingdom. Curr. Opin. Plant Biol. 55, 100-108. doi: 10.1016/j.pbi.2020.04.004

Winkel, B. S. J. (2006). "The biosynthesis of flavonoids" in The Science of Flavonoids. ed. E. Grotewold (New York, USA: Springer Scienceand Business Media, Inc), 71-96.

Xu, F., Cheng, H., Cai, R., Li, L. L., Chang, J., Zhu, J., et al. (2008). Molecular cloning and function analysis of an anthocyanidin synthase gene from Ginkgo biloba, and its expression in abiotic stress responses. Mol. Cells 26, 536-547.

Xu, F., Li, L., Zhang, W., Cheng, H., Sun, N., Cheng, S., et al. (2012). Isolation, characterization, and function analysis of a flavonol synthase gene from Ginkgo biloba. Mol. Biol. Rep. 39, 2285-2296. doi: 10.1007/s11033-011-0978-9

Yan, Y., Chemler, J., Huang, L., Martens, S., and Koffas, M. A. (2005). Metabolic engineering of anthocyanin biosynthesis in Escherichia coli. Appl. Environ. Microbiol. 71, 3617-3623. doi: 10.1128/AEM.71.7.3617-3623.2005

Conflict of Interest: The authors declare that the research was conducted in the absence of any commercial or financial relationships that could be construed as a potential conflict of interest.

Publisher's Note: All claims expressed in this article are solely those of the authors and do not necessarily represent those of their affiliated organizations, or those of the publisher, the editors and the reviewers. Any product that may be evaluated in this article, or claim that may be made by its manufacturer, is not guaranteed or endorsed by the publisher.

Copyright (c) 2021 Busche, Acatay, Martens, Weisshaar and Stracke. This is an open-access article distributed under the terms of the Creative Commons Attribution License (CC BY). The use, distribution or reproduction in other forums is permitted, provided the original author(s) and the copyright owner(s) are credited and that the original publication in this journal is cited, in accordance with accepted academic practice. No use, distribution or reproduction is permitted which does not comply with these terms. 